\title{
Handover management in GSM cellular system
}

\author{
Jahangir khan \\ School of computer science \\ PAF-KIET, Pakistan Air Force Base \\ korangi Creek Karachi 75190 \\ Pakistan
}

\begin{abstract}
Handover mechanism is extremely important in cellular network because of the cellular architecture employed to maximize spectrum utilization. Handover is the procedure that transfers an ongoing call from one cell to another as the user's moves through the coverage area of cellular system. One way to improve the cellular network performance is to use efficient handover prioritization schemes when user is switching between the cells. In this paper I have presented an analytical framework that can enhance considerably the handover call mechanism in wireless network. Some advance schemes namely, guard channels, call admission control and handover queuing are discussed. All these of prioritiztaions schemes have a common characteristic reducing the call dropping probability at the expense of increased call blocking probability. Efficnet prioritiztaion scheme accomadetes a number of new calls while guaranates the quality of sevice(QOS) of hanover call. This idea is based on the neighbouring cells have an ovelapping (the area served by more than one cell) coverage area. Furthermore cell overlap and load balancing scheme is proposed to enhance the GSM cellular capacity using an overlapping coverage area. Capacity enhancement is achieved by balancing the load in neighboring cells.
\end{abstract}

\section{General Terms}

Call Admission Control, Signaling Connection Control Part, Received Signal Strength, Location Area Code, Cell Identify. Keywords

GSM, QOS, Handover management, soft handoff.

\section{INTRODUCTION}

We Due to rapid change in technology the demand for better and faster cellular communication also increases. This growth in filed of cellular communication has led to increase intensive research toward and development toward cellular system. The main reason of this growth is newly concept of mobile terminal and user mobility. The main characteristics of cellular communication system offer user maximum freedom of moment while using cell phones (mobiles). A cellular network is made up of numbers of cells (or radio cells). Each cell is allocated a band of frequencies and served by base station consisting of transmitter, receiver and control unit. Adjacent cells are assigned different frequencies to avoid interference or cross talk [7]. As more customers use the cellular network with single base station traffic may be build up so there are not enough frequency bands assigned to a cell to handle its calls. An approach can be used to cope with this situation to use the same radio frequency can be reused in different area for a completely different transmission [25]. The degree of reuse determined by how apart cells must be reuse the same frequency is depending upon the actual implementation of the radio link. The reuse of frequencies in different cells is a form of space division multiple access and it requires that location of each mobile agent to be known this is provided through a service known location management or mobility management. The obstruction in cellular network involves the problem when a mobile user travels from one cell to another during a call. As adjacent cell do not use the same radio channels, a call must be transferred from one radio channel to another when a user crosses the line between the adjacent cells. The process of handover takes place that transfer an ongoing call from one cell to another cell as the user moves through the coverage area of a cellular network. In handover process cellular network automatically transfer a call from one radio channel to another radio channel while maintaining quality of services (QoS) of a call [14]. Handover mechanism is extremely important in mobile network because of the cellular architecture employed to maximize spectrum utilization. The number of cell boundaries increases because smaller cells are deployed in order to meet the demand of increased capacity. Each handover require network resources to route the call to next base station. If handover does not occur at right time the QoS may be drop below an adequate level and connection will be dropped [9]. There are several different reasons needed to be known to determine whether a handover is required. The signal strength of the base station, along signal strengths of the surrounding stations. In addition the availability of the channels also needed to be known. The mobile monitors the strength of the base stations, but only the cellular network knows the status of channel availability and the network make the decision about the handover.

The main goal of this research is to investigate the GSM handover research issues and developing schemes which can handle handovers traffic in order to support on-going calls when mobile users are switching between base stations. The final outcomes of the research will theoretical results to convince the users as possible. There are several sub goals in the research while achieving the main goal. The sub goals of the research are to study different handover mechanisms that reduce the resources utilizations of the GSM network while reducing the dropping the possibilities of the user calls. To introduce new concepts and handover schemes for enhancing and optimizing GSM wireless network performance. To provide effective methods for evaluating GSM handover mechanisms as well as cellular network performance.

The paper is organized as follows. In Section 2, I describe evaluation of cellular networks which leads for Global system for 
mobile communication, Cell Overlapping and Load Balancing Scheme (Proposed Scheme) and also conventional hand over mechanism. In section 4 I analyzed the hand over management in GSM cellular networks and lastly in Section 5 I conclude the paper.

\section{EVALUATION OF CELLULAR NETWORKS}

The cellular network has gone through three generations. AMPS the first generation of cellular networks are analog base which is the current standard of U.S cellular network. To increase network capacity new technologies TDMA (time division multiple access) and CDMA (code division multiple access) were used in GSM (global system for mobile communications) second generation to accommodate more mobile terminals. The third generation cellular phone providing high speed data transmission with voice transmission. The following section illustrates the cellular network generation in general [34].

\subsection{Advanced Mobile Phone System (AMPS)}

The advanced mobile phone system (AMPS) is the first commercial cellular system. AMPS was invited by Bell labs and introduces in the early 1980 based on the radio telephone system. AMPS mobile system used analog transmission for voice conservation. Since AMPS uses FDMA (frequency division multiple access) which means each conservation is divided by using different channel. Two $25-\mathrm{MHz}$ bands are allocated to AMPS one for transmission from the base station to mobile terminal (869-894 MHz), the other for transmission from mobile terminal to base station $(824-849 \mathrm{MHz})$. The channels are spaced by $30 \mathrm{kHz}$ away from each other which leave 416 channels per operator. Twenty one channels are allocated for control and 395 for voice [8]. AMPS cellular network maximized the cellular concept by frequency reusing, handover and roaming capabilities. As AMPS cellular system is purely analog using FM; therefore digital traffic is supported only by using modem that convert the digital data into voice. In first generation cellular network a cell covers number of channels. At a time a channel is allocated to one user. Limitation associated with AMPS includes limited spectrum, low calling capacity, poor data communications and minimal privacy [25].

\subsection{GSM (Global System for Mobile Communications)}

GSM is a second generation digital cellular system. Digital transmission was used rather than analog transmission in order to improve transmission quality, system capacity, and coverage area. GSM works on three frequencies $900 \mathrm{MHz}, 1800 \mathrm{MHz}$ and $1900 \mathrm{MHz}$. To make efficient use of frequency bands GSM networks uses combination of FDMA (frequency division multiple access) and TDMA (time division multiple access). GSM operators have set up roaming agreement with foreign operator which help users to travel abroad and use their cell phones [10].

\subsubsection{GSM Network Architecture}

The general architecture of GSM network is shown in figure 1 . The GSM system consist of several functional elements including mobile switching centers (MSC), base stations (BSC) with associated base transceivers (BTS), an operation and maintenance centre (OMC) and gateway MSC.GSM mobile terminal or mobile stations communicates across the Um interface, known as the air interface, with a base BTS in the small cell in which the mobile unit is located. This communication with a BTS takes place through the radio channels. The network coverage area is divided into small regions called cells. Multiple cells are grouped together form a locations area (LA) for the mobility management.

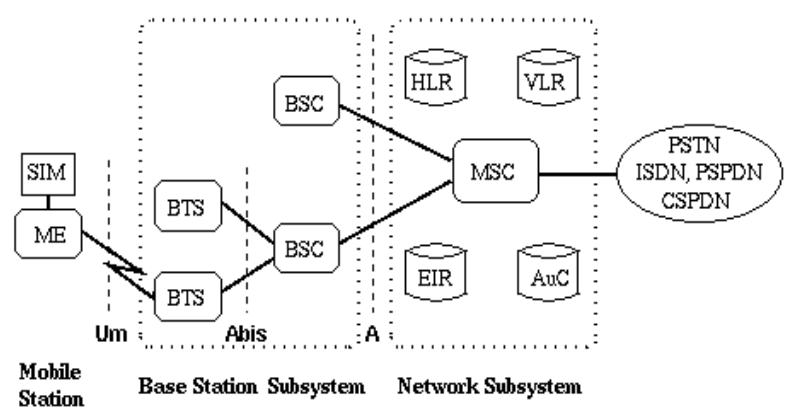

Figure 1. GSM Network Architecture

BSC are connected are connected to MSC through dedicated line or radio communication link. The BSC reserves radio frequencies, manages the handover of mobile station from one cell to another with in the BSS (base station subsystem). MSC interface to the PSTN (public switched telephone network) is called the gateway MSC. MSC incorporate functions including home location register (HLR), visitor location register (VLR), authentication register $(\mathrm{AuC})$ and equipment identity register (EIR) [23]. The HLR and VLR together with MSC provide the call routing and roaming capabilities of GSM. The HLR stores information both permanent and temporary about each of the mobile station that belongs to it. The VLR register maintains information about mobile station that is currently physically in the region covered by MSC. VLR becomes important when user leaves the area served by his home MSC. The two registers are used for authentication and security purpose. The EIR is a database that contains a list of all valid mobile equipment on the network, where each mobile station is identified by its international mobile equipment identity (IMEI). It helps in security and prevents uses of network by mobile station that haven been approved. The AuC holds the authentication and encryptions keys that are stored in each user SIM card for authentication and encryption over radio channel [17].

\subsection{GSM Radio Interface}

The ITU-T (International Telecommunication Union) allocated the $890-915 \mathrm{MHz}$ frequency for uplink transmission and 935-960 $\mathrm{MHz}$ for downlink transmission to GSM network in Europe. Due to the limited radio bandwidth GSM utilizes a combination of FDMA and TDMA called Multi-Carrier TDMA to access the available radio spectrum. Older standards of mobile system use only FDMA. In FDMA system one specific frequency is allocated 
for every user during a call. FDMA overload network in case of high user demand. The overload problem which leaded mobile communications systems by defining two dimensional access scheme [17]. Where TDMA allows several users to share the same frequency channel by dividing the signal into different timeslots. This allows multiple stations to share the same radio frequency channel while using one part of its bandwidth. GSM system always uses TDMA with in FDMA structure. TDMA major advantage is that the radio part of mobile needs to listen and from its own timeslot. All the time mobile can transmit measurements on the network detecting surrounding transmitters on different frequencies. This makes handover simpler and safe.

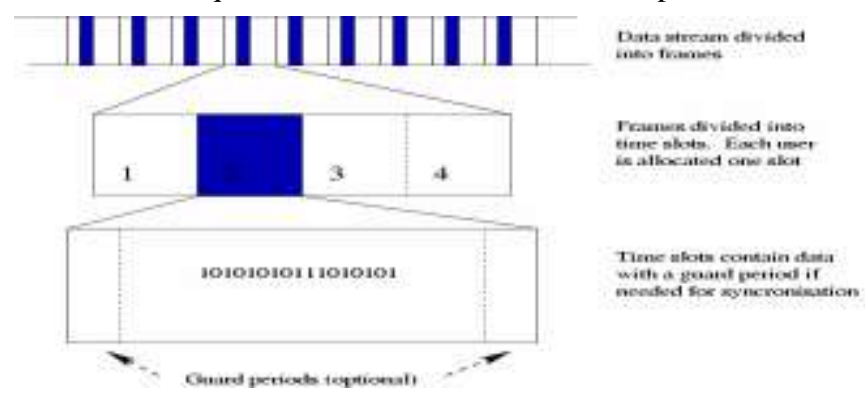

Figure 2. TDMA Frame Structure.

The above figure shows the TDMA frame structure showing a data stream divided into frames and those frames divided into timeslots [29].

\subsubsection{GSM Burst and its Structure}

All carrier frequency is divided according to time slot using TDMA scheme. Each carrier frequency is further divided into a $120 \mathrm{~ms}$ multiframe which is made up of 26 frames. In which two frames are used for control purpose while remaining 24 frames are used for traffic [23].

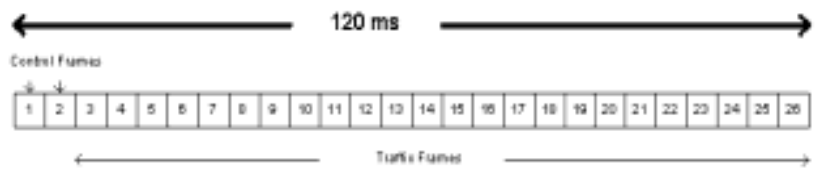

Figure 3. Structure of a Multiframe

Each frame is further divided into 8 burst and each of the 8 bursts is assign to a single to single user. In TDMA system burst is a unit of time and every burst ends in $0.577 \mathrm{~ms}$.

\begin{tabular}{|l|c|c|c|c|c|c|c|}
\hline Burs & Borst & Burst & Burst & Burt & Burst & Burs & Burst \\
\hline
\end{tabular}

Figure 4. Structure of a Frame

\subsection{GSM Physical and Logical Channels}

In GSM a number of logical channels multiplexed onto physical channels, which allows system to run multiple activates in parallel which doest not require to use dedicated every slot transmission. Thus the logical channels improve the physical channels reuse capabilities to high level. Logical channels are linked on the physical channels so called laid over grid of physical channels. Another important feature of logical channels that during a call MSC sends its signal periodically at the same
TDMA frame on the same time slot to BTS and same from the BTS to MSC in reverse direction [22], GSM channels are mainly group into two categories traffic channels and control channels. Traffic channels carry speech and data information. A traffic channel using 26 TDMA frames in this standard traffic channels for uplink and downlink are separated by 3 burst. In other words traffic channels use 1 time slot in each traffic frame. Control channels deals with network management and channel maintenance tasks. Control channels involve system signaling and synchronization data for control procedure such as location registration, mobile station synchronization, paging, random access between base station and mobile station. There main categories of control channels are defined [31].

\subsubsection{BCH (Broadcast Channel)}

Broadcast control channel is a downlink channel that is used by the BTS to broadcast information to mobile station and inform them about the incoming calls. It is required in initial to provide a time slot for a call. BCCH broadcast general information such power control parameters, access methods, network parameters etc required to set up calls. The three broadcast channels are defined. First broadcast channel is FCCH (Frequency Correction Channel) Provides MSC with the frequency reference of the system to allow synchronization with the network and frequency drift correction. Second broadcast channel SCH (Synchronization Channel) Provides frame synchronization for MSC and identification of BSC. The synchronization channel transmits the sequence that is needed for link quality estimation and equalization. Third broadcast control channel $(\mathrm{BCCH})$ which is also downlink channel that is used by BT to broadcast information to mobile station and inform them about the incoming calls. It is required in initial to provide a time slot for a call. BCCH broadcast general information such power control parameters, access methods, network parameters etc required to set up calls [6].

\subsubsection{Common Control Channel (CCCH)}

The common control channel is a combination of common control channels that is used between MSC and BSC before a dedicated control channel is allocated. There are three downlink paging, access grant and cell broadcast channels and one random access uplink channel. Paging channel (PCH) is activated for selective addressing of a mobile station during a connect request from the network. Random access channel ( $\mathrm{RACH})$ is transmitted by mobile station as uplink and to access the network and request channel capacity form the BSC to establish a connection. The access grant channel $(\mathrm{AGCH})$ channel is transmitted by the BSC in response of random access from MSC. According to the call setup mechanism selected by network operator is allocated for call. Cell broadcast channel $(\mathrm{CBCH})$ channel containing broadcast messages information about the traffic information etc. [6].

\subsection{Frequency Reuse Distance and Cluster Size}

A GSM cellular network is made of number of radio cells or cells served by fixed base station. These cells are used to cover different areas to provide radio coverage over wider area. These radio cells are combined into clusters and each frequency is used 
once per cluster. The capacity in cellular network can be increased comes form the fact that the same radio frequency can be reused in different area for completely different transmission in a regular way. The ruse of frequencies enables a cellular system to handle huge number of calls with limited numbers of channels. GSM cellular layout typically involves the frequency reuse factor which is inversely proportional to $\mathrm{K}$ (where $\mathrm{K}$ is number of cell per cluster). The value of $\mathrm{K}$ is 7 for TDMA system. The co- channel interference is serious problem in this scheme while adjacent co-channel interference is not a big problem. Where $\mathrm{R}$ is the radius of the cell and $\mathrm{D}$ is the distance from the center of the cell to its neighbor using the same frequency. The minimum separation between two cells using the same frequency so that the two cells don't not interference with each other can be calculated by $D / R=\sqrt{ } \overline{3} K$ as shown in the following figure 5. [5].

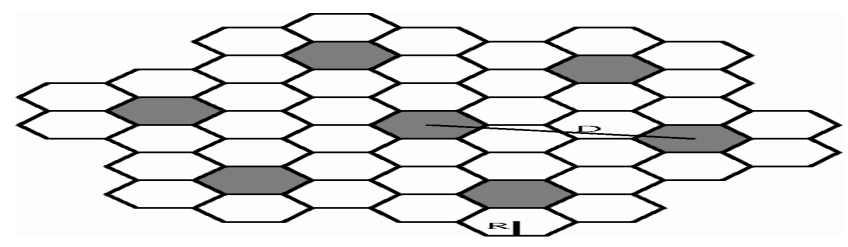

Figure 5. GSM Cellular Layout for Frequency Reuse

In TDMA the engineers should also make sure that the power level at the cell boundaries is weak enough not to drop over into adjacent cells. By placing a base station at the center and the interfacing stations on the perimeters of circle radius D we can simulate the received signal with co-channel interference at mobile coming from its own with interfering base station known as forward CCI (co-channel interference). The simulation results show that the shown interference level grows near the cell boundaries and its level changes with cell radius as in the figure 6.

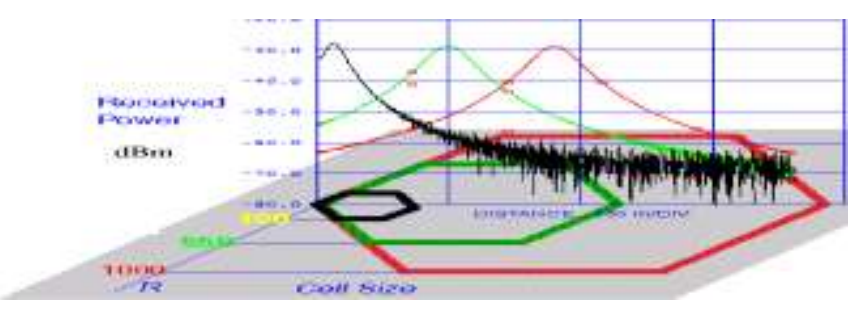

Figure 6. Received Signal Power for three Different Values of D

\subsection{GSM Handover Procedure}

Handover is the procedure that transfers an ongoing call from one cell to another as the user's moves through the coverage area of cellular system. The purpose of the handover procedure it to preserve ongoing calls when the mobile station moving from one cell to another. In GSM measurements reports to perform the handover, which is made by the serving BSC which has no direct knowledge of the radio quality. These measurements reports contain the radio signal quality of the downlink from the BTS to MSC of the call and up to five neighboring cells. The serving BTS measures the uplink from the MSC to BTS radio signal quality of the call and forward in the measurements reports. The information in the measurements reports the BSC is able to decide weather a handover to another cell is needed. These measurements reports are periodically transmitted from the MSC to BSC on the SACCH channel assigned to each communication for every connection. The repetition duration of the SACCH produces a fixed time grid of $480 \mathrm{~ms}$ in the measurements reports. The figure 7 . shows the measured RXLEVs from the BTS and from a neighbor to the measurements reports submitted during a call are shown [9].

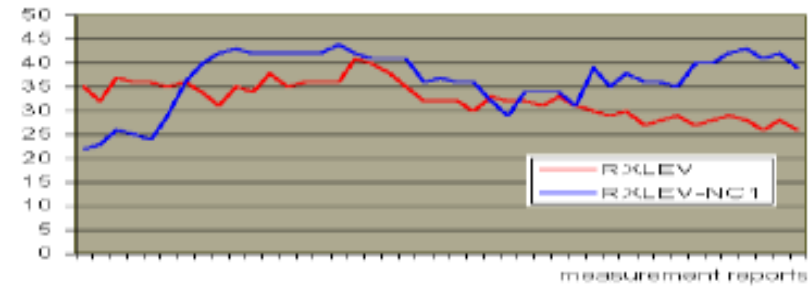

\section{Figure 7.Signals Strength Measurements Handover} Procedure

In the above figure horizontal axis shows the measurements reports. In many cases these measurements reports takes place to take handover procedure in a way to avoid shortcomings. A connection is continuously measured and evaluated by the respective base station and MSC. Handover is based upon that evaluation. As the mobile users leaves the coverage area of the one base station must obtain coverage from the neighboring station in order to keep the connection keep on. Cut off connection or drop call are not acceptable at any level during the call. Handover take places when the traffic level of cell reaches to high level or when neighboring are being underutilized. The figure 8. summarizes the handover causes showing the percentage of different reasons of handover [9].

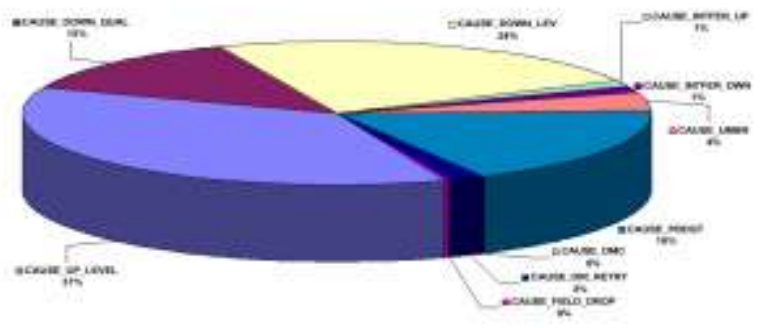

Figure 8. Handover Causes

According to the above pie-chart the main reason for handover initiations is the uplink, downlink level with $61 \%$. Other reasons are; power budget is $18 \%$, downlink quality $13 \%$, umbrella-cell handover, interference, directed retry and OMC shortcomings. In GSM cellular network a number of different performance metrics is commonly used to make the handover decision. These performance metrics gives foundation after many research into the microcellular handover and overlaid system handover as Cell Blocking Probability, Call Dropping Probability, Call Completion Probability, Probability of Unsuccessful Handover, Handover Blocking Probability, Rate of Handover, Interruption Duration and Handover Delay.

\subsubsection{GSM Handover Initiation}

Handover initiation is the process of deciding when a request to a handover. Handover is based on received signal strength (RSS) 
from the current base station and the neighboring base station. The figure 9 shows a mobile station is moving form one BTS (named BTS1) to another BTS (named BTS2). The RSS of BTS1 decreases as the mobile station moves away and increases as the mobile station get closer to the BTS2 as a result of the signal propagation. Form the below figure 9. We examine various approaches to handover initiation as in the forms

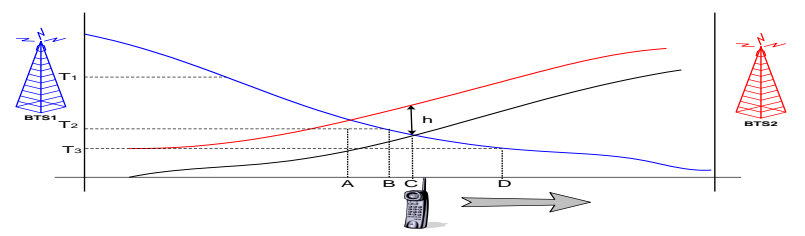

Figure 9. Movement of the MS between two Adjacent BTS for Potential Handover, Relative Signal Strength, Relative Signal, Strength with Threshold, Relative Signal Strength with Hysteresis, Relative Signal Strength with Hystersis and Threshold, Prediction Approaches.

\subsubsection{GSM Handover Type}

There are different categories of GSM handover which involves different parts of the GSM network. Changing cells within the same BTS is not complicated as the changing of the cell belonging to different MSC. There are mainly two reasons for this kind of handover. The mobile station moves out of the range station or the antenna of BTS respectively. Secondly the wire infrastructure the MSC or the BSC may decide that the traffic in one cell is too high and move some to other cells with lower load. These are the main reasons that initiate different kinds of handover. Following are the different kinds of handover and their details.

Intra-cell BTS Handover: The terms intra-cell and intra BTS handover are used both for frequency change. There is a slight between them but usually they are considered the same. The term intra-cell handover in not real as it deals with the frequency change of a going call. The frequency change occur when the quality of the communication link degrading and the measurements of the neighboring cells better than the current cell. In this situation the BSC which controls the BTS serving the MSC order the MSC and BTS to switch to another frequency which offers better communication link for the call. The communication link degradation is caused by the interference as the neighboring cell using the same frequencies and its better to try another channel. In the intra BTS handover cell involved are synchronized. In the synchronization the MSC sends four HND_ACC messages to the serving BTS. The HND_ACC message is only one byte long and contains the handover reference. The BSC send the HND_CMD to initiate the intra BTS handover. The BSC HND_CMD the time slot and the new channel. The connection is established between the BSC and MSC by exchanging these messages. After these messages exchanged MSC receives HND_PREF message that the handover is performed by the $\mathrm{BSC}$ and then $\mathrm{BSC}$ requests $\mathrm{BTS}$ to release the resources that is no longer used. This synchronized handover saves resources and faster than non synchronized handovers [26].

Intra-BSC Handover: The intra-BSC handover is performed when the MSC changes the BTS but not the BSC. The intra-
BSC handover is entirely carried out by the BSC, but the MSC is notified when the handover has taken place. If the targeted cell is in different location area then the MSC needs to perform the location updates procedure after the call. In the intra-BSC handover both synchronized and non synchronized handover are possible. The figure $\mathrm{X}$ shows the intra-BSC situation.

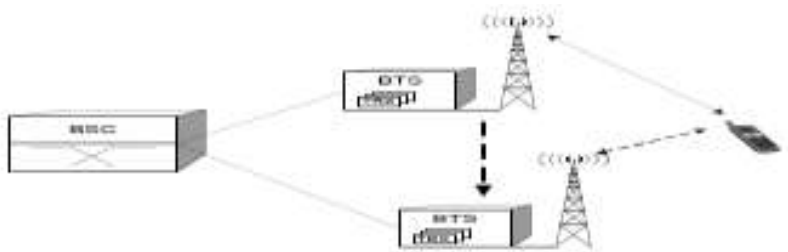

Figure 10. Intra-BSC Handover

In the intra-BSC handover the BSC send HND_CMD message which contain the time slot, the frequency of the new channel and how MSC shall identify itself on the new channel. And at the same time BTS send PHYS_INFO message and wait for the SABM (Set Asynchronous Balance Mode) from the MSC. When the MSC receives PHYS_INFO message then send SABM in order to establish LAPD for mobile. When the BTS receives the SABM it sends an empty as an acknowledgement to the BSC. Now the MSC only receive the information that the handover was performed by the BSC and BTS releases the used radio resources [26].

Intra-MSC Handover: In the intra-MSC handover when the BSC decides that handover is required but the targeted cell is controlled by different BSC then it needs assistance form the connected MSC. In comparison to the pervious handover discussed the MSC mandatory for this kind of handover. Responsibilities of the MSC do not include processing the measurements of the BTS or MSC but to conclude the handover. This kind of handover can be other intra-MSC or Inter-MSC. In the intra-MSC handover the targeted cell is allocate in different BSC connected by the same MSC. The MSC contacts the targeted BSC for allocation of the required resources and inform the BSC when they are ready. After the successful resources allocation the MSC instructed to access the new channel and the call is transferred to the new BSC as shown in the figure 11. [26].

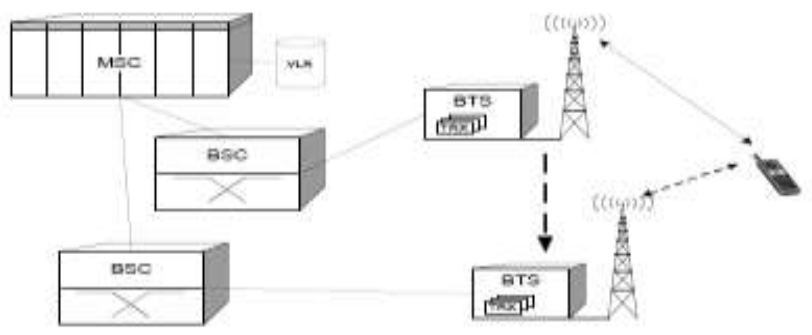

Figure 11. Intra-MSC Handover

Inter-MSC Handover: The inter-MSC handover is performed when the two cells belonging to different MSC in the same system. In the inter-MSC handover the targeted cell is connected is connected to different MSC (named as MSC-B) than the one currently serving the call MSC (named as MSC-A). Figure shows $\mathrm{XII}$ the inter-MSC handover scenario. 


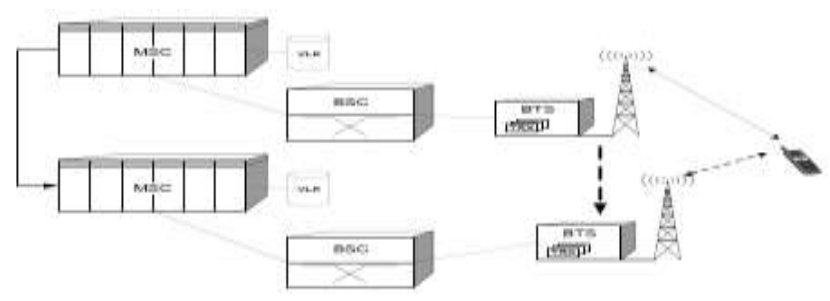

Figure 12. Inter-MSC Handover

When BSC-A determines that a handover is into another area its sends HND_RQD to its connected MSC-A. HND_RQD contain information the CI (cell identify) and the LAC (location area code) belonging to another MSC area. After identifying the correct neighbors MSC sends prepare handover to MSC-B. The VLR of the MSC-B assign the temporary handover number and passes the HND_REQ to the target BSC-B. In the response of HND_REQ message BSC-B sends HND_REQ_ACK if the resources are available to MSC-B. The MSC-B forward to prepare for handover by sending BSSAP message back to MSCA. In the response MSC-A sends IAM which contains the handover number between MSC-A and MSC-B and after getting the correspondent ACM from MSC-B, MSC-A sends HND_CMD message to MSC-B to perform the handover to the target BTS. The BSC-B sends HND_DET that HND_ACC was received from the MSC. The HND_DET message is forwarded in a MAP to MSC-A. Now the traffic channel is established between MSC-A and MSC-B, and the transport of the payload is carried to MSC-B, and the handover number is released by VLR in MSC-B. By receiving HND_CMP at MSC-B is signaled to MSC-A in a MAP message in Send End Signal. This triggers MSC-A to send CLR_CMD message to BSC-A to release the radio resources [28].

\subsubsection{GSM Handover Measurements}

The mobile station makes measurements which are used in triggering of the handover and in the evaluation of the handover candidate cell. This makes the measurements as essential part of the handover process. In order to make efficient handover these measurements should be refreshed very fast as possible. The mobile station measures the system parameter continuously and the level of the neighboring cells and sends this information to the network so that the decision for the handover is available to network all the times. These measurements repots from the mobile station is carried on the SACCH signaling channel after every $0.48 \mathrm{sec}$ but a minimum once per second. In capacity the SACCH channel is error free which means that the measurements reporting is perfect almost. This measurements report contains parameters that define the current network connection, the radio conditions of the neighboring cells and the targeted cells to handover. In GSM one measurements message send from a mobile station to the BTS every $0.48 \mathrm{sec}$ contains the signal level up to 6 neighboring cells. However a mobile station may pre-synchronize with more than 6 neighboring cells. In this case the measurements corresponding to the 6 cells but it receive best are reported to the BTS. The measurements of the neighboring cells is more difficult because a mobile station mush establish which neighboring cell it can receive and divided the measurements times among those cells capable of receiving. The possible measurements times are between the transmission and reception of a burst of the traffic channel. The figure 13. Shows time which is not suitable for measurements of the channel of neighboring cells.

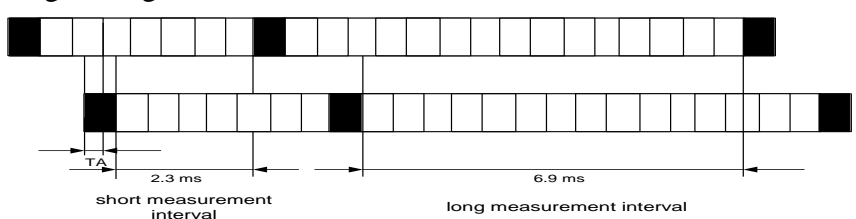

Figure 13. GSM SACCH Block

When there is discontinuous between the transmissions then the GSM measurements become inaccurate. To overcome these problems the power control and handover settings should be set more accurate [20].

\section{GSM CALL HANDLING MECHANISM}

In this section a couple of enhancements which can improve the performance of GSM handover algorithms are presented and studied.

\subsection{Conventional Handover Mechanism}

In GSM cellular network both the mobile station and the BTS regularly measures the radio signal strength. The mobile station transmits its measurements reports continuously to the BTS. If the BTS detects a decrease in radio signal under a minimal level $\mathrm{d}_{\text {urge, }} \mathrm{cf}$ it initiates a handover request as shown in figure 14 . The BTS then informs the BSC about the request, which then verifies if it is possible to transfer the call into a new adjacent cell. Actually the BSC checks weather a free channel is available in the new adjacent cell or not. In this situation the BSC does not differentiate between the channel requests either for fresh call or handover. If a free channel is available in the new adjacent cell then handover request can be satisfied, and the mobile station switch to new cell. If there is no free channel in the adjacent cell then it increases the dropping probability of handover call. The drawback of this handover procedure is the fact that the handover request for channel is same as used for fresh calls cf. In conventional handover mechanism is very problematic from the users quality of service perspective, since user can much prefer block a fresh call rather than to be dropped a call in the middle of transmission [19].

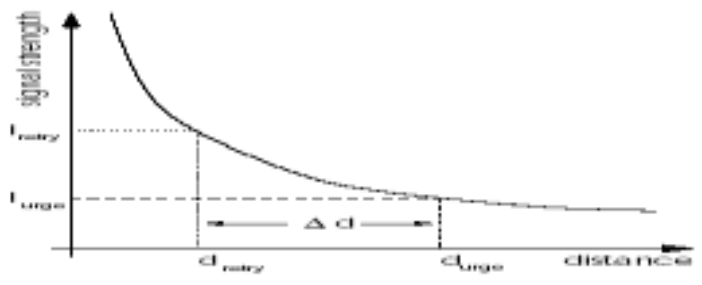

Figure 14. Signal Levels for Handover

\subsection{Channel Carrying Handover Mechanism}

The channel carrying mechanism allows a mobile station to carry its current channel from one cell to another when it moves across the boundaries under specific conditions. The channel carrying mechanism using a linear cellular system model in which cells or BTS are arranged in linear configuration with minimum reuse distance $r$ as shown in the figure 15 . Suppose $\mathrm{N}$ be the total number of channels available for use in cellular system. Two 
cells can use the same set of channel as they are apart by distance r.

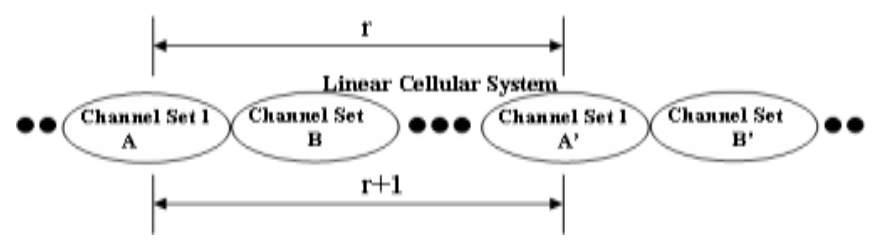

Figure 15. $r$ and $(r+1)$ Channel Carrying

To avoid the co-channel interference an advance solution is proposed in which the distance of identical sets of channels is increased to $r+1$ instead of $r$. The distance $r$ is the minimum reuse distance or reuse factor. According to the figure the total number of available channels in each cell is now reduced by amount of $\mathrm{N} / \mathrm{r}+1$ where $\mathrm{N}$ is the total number of available channels. In typical situation where the smaller the reuse distance the more amounts of channels is to be lost. The channel carrying mechanism does not require the complex power control or global channel coordination which simplifies its implementation. Handover request are greatly favored over new calls compared to the Conventional handover mechanism. The main drawback of this handover procedure is not suitable for metropolitan environment due to the great amount of channels lost [18].

\subsection{GSM Handover Prioritization Schemes}

Different ideas and approaches are proposed to reduce the handover dropping probability. One approach is to reduce the handover failure rate is to prioritize handover call over new calls. Handover prioritization schemes have a significant impact on the call dropping probability and call blocking probability. Such scheme permits high utilization of bandwidth while guaranteeing the quality of service of handover calls. Basic method of handover prioritization schemes are guard channels (GC), call admission control (CAC) and handover queuing schemes. Some times these schemes are combined together to obtain better results [27].

\subsubsection{Guard Channel Prioritization Scheme}

The guard channel scheme was introduced in 80 s for mobile cellular systems. However the guard channel scheme are still used in telecommunications with the name of Cutoff Priority Schemes. GC scheme improving the probability of successful handover by simply reserving a number of channels exclusively for handover in each cell. The remaining channels can be shared equally between handover and new calls. GC are established only when the number of free channels is equal to or les than the predefined threshold $\mathrm{g}$ as shown in figure 16. In this situations fresh calls are bypassed and only handover request are served by the cell until all channels are occupied. The GC scheme is feasible because new calls are less sensitive to delay than the handover calls [15].

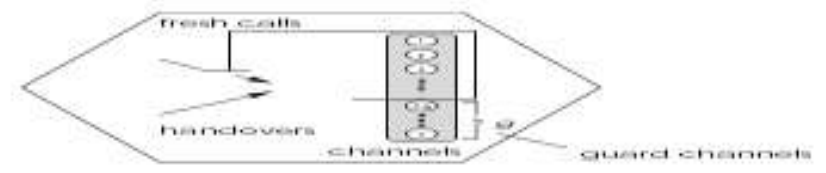

Figure 16. Guard Channels for Handover Request
If we consider a cellular network with $\mathrm{C}$ the total number of channels in a given cell. According to GC scheme reserve channels for handover are $\mathrm{C}-\mathrm{T}$ where $\mathrm{T}$ is the predefined threshold. The GC will not accept any new call until the channel occupancy goes below the threshold. Suppose the arrival of new and handover call is denoted with $\lambda$ and $v$ respectively. The call holding and call residency for both call is exponentially distributed with $1 / \mu$ and $1 / \eta$ respectively. The total traffic can b e calculated as $\rho=(\lambda+v) /(\mu+\eta)$. Figure 17 . Shows the state transition diagram of guard channels.

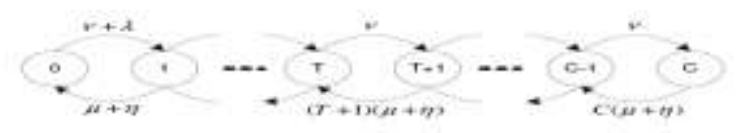

Figure 17. State Transition Diagram of Guard Channels

Therefore according to the cell occupancy by Markov chain it is straight forward to derive the steady state probability $\mathrm{P}_{\mathrm{n}}$ that $\mathrm{n}$ channels are busy [10].

$$
P_{n}= \begin{cases}\left(\frac{\rho^{n}}{n !}\right) P_{0}, & 0 \leq n \leq T \\ \rho^{T}\left(\frac{\nu^{n-T}}{n !}\right) P_{0}, & T \leq n \leq C\end{cases}
$$

Where

$$
P_{0}=\left[\sum_{n=0}^{T} \frac{\rho^{n}}{n !}+\rho^{T} \sum_{n=T+1}^{C} \frac{\nu^{n-T}}{n !}\right]^{-1}
$$

$\mathrm{P}_{\mathrm{b}}=\sum^{\mathrm{C}}{ }_{\mathrm{n}=\mathrm{T}+1} \quad \mathrm{P}_{\mathrm{n}}$ and $\mathrm{pf}=\mathrm{Pc}$. In fact, there is a tradeoff between minimizing $\mathrm{Pd}$ and minimizing $\mathrm{Pb}$. If the number of channels is static chosen then the admission call control fails to satisfy the specified Pd. The static channels reservation shows results poor utilization of bandwidth [10].

\subsubsection{Call Admission Control Prioritization Scheme}

The call admission control scheme refers to the task of deciding weather new call requests are admitted into the network or not. In the CAC the arrival of new call are estimated continuously and if they are higher than the predefined threshold level then some calls are restricted (blocked) irrespective of weather a channel is available or not to decrease the probability of handover calls. In the CAC both the new and handover calls have to access to all channels. If a new call that is generated in cell cannot find an idle channel the call is discarded immediately. There is no queue provided for the new calls to wait [1]. The CAC scheme can be classified into different schemes that consider the local information like (the amount of unused bandwidth in cell where the user currently resides), remote information (the amount of unused information bandwidth in the neighboring cells) or local or remote information to determine weather to accept or reject a call. CAC based on knowledge of both network and user characteristics, keeps the track of available system capacity and accommodates new call request while ensuring quality of service for all existing users. Decisions in CAC are performed in each $\mathrm{BSC}$ in a distributed manner and there is no central coordination. 
The CAC scheme can be evaluate on the basis of Efficiency, Fairness, Stability and Flexibility [32].

\subsubsection{Handover Queuing Prioritization Schemes}

Queuing handover call prioritization scheme queues the handover calls when all the channels are occupied in the BSC. When a channel is released in the BSC, it is assign to one of the handover call in the queue. The handover queuing scheme reduces the call dropping probability at the expense of the increased call blocking probability and decrease in the ratio of carried to admitted traffic since new call are not assign a channel until all the handover request in the queue are served [21]. In the handover queuing schemes when the received signal strength of the BSC in the current cell reaches to certain define threshold the call is queued from service a neighboring cell. A new call request is assigned a channel if the queue is empty and if there is at least of free channel in the BSC. The call remains queued until either a channel available in the new cell or the power by the base station in the current cell drops below the receiver threshold. If the call reaches the receiver threshold and no free channel if found then the call is terminated. Queuing handover is possible due to the overlap regions between the adjacent cells in which the mobile station can communicate with more than one base station. This makes provision of the queuing the handover requests for certain time period equal to the time of the mobile host existence in the overlapping area [12]. Queuing is effective only when the handover requests arrive in groups and traffic is low. First in first out (FIFO) scheme is the most common queuing scheme where the handover requests are ordered according to their arrival. To analyze this scheme it is necessary to consider the handover procedure in more detail. By assuming the FIFO queuing strategy and infinite queue size at the base station is assumed as shown in figure.17,

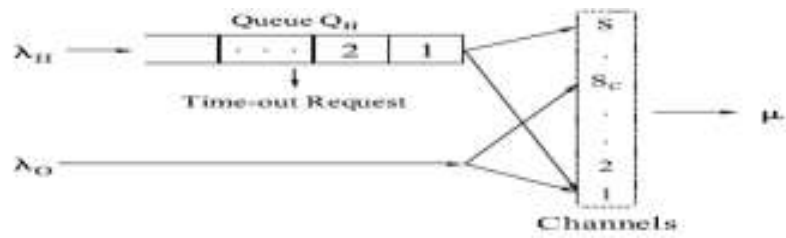

Figure 17. Priority Queue System Model for Handover Call

The handover of the mobile station depends on the system parameters such as moving speed, the direction of the mobile station and the cell size. Suppose the state $i(i=0,1,2 \ldots, \infty)$ of a cell as the sum of the channels being used and the number of the handover call request in the queue. Then it is clear form the Markov chain that $i$ one- dimensional. The blow figure shows the sate transition diagram of the cells [33].

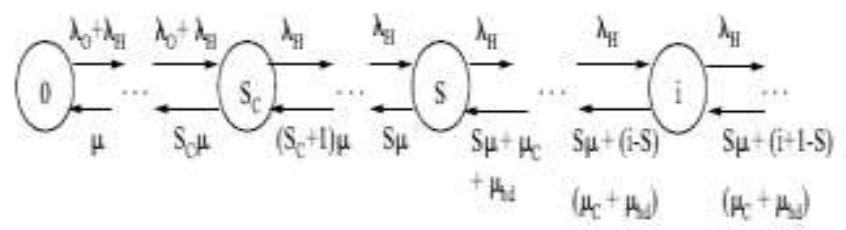

Figure 18. State Transition Diagram

From the above figure the equilibrium probabilities are related to each other through the following equations.

$$
\begin{array}{ll}
i \mu P(i)=\left(\lambda_{O}+\lambda_{H}\right) P(i-1) & 0 \leq i \leq S_{C} \\
i \mu P(i)=\lambda_{H} P(i-1) & S_{C}<i \leq S \\
{\left[S \mu+(i-S)\left(\mu_{C}+\mu_{h-\text { dwell }}\right] P(i)=\lambda_{H} P(i-1)\right.} & S<i \leq \infty
\end{array}
$$

Then the steady state probability is found as follow according to the Markov chain

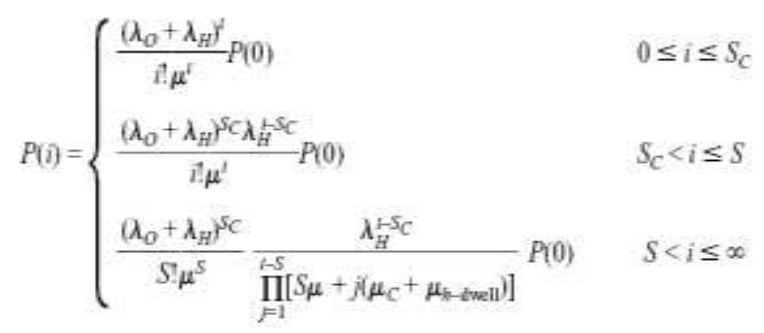

Where

$$
\begin{aligned}
P(0)= & \left\{\sum_{i=0}^{S_{C}} \frac{\left(\lambda_{O}+\lambda_{H}\right)^{t}}{i ! \mu^{i}}+\sum_{i=S_{C}+1}^{S} \frac{\left(\lambda_{O}+\lambda_{H}\right)^{S_{C}} \lambda_{H}^{i-S_{C}}}{i ! \mu^{i}}\right. \\
& \left.+\sum_{i=S+1}^{\infty} \frac{\left(\lambda_{O}+\lambda_{H}\right)^{S_{C}}}{S ! \mu^{S}} \frac{\lambda_{H}^{i-S_{C}}}{\prod_{j=1}^{i-S}\left[S \mu+j\left(\mu_{C}+\mu_{n-\text { dwell }}\right)\right]}\right\}^{-1}
\end{aligned}
$$

Therefore the blocking probability Bo for an originating call is

$$
\begin{gathered}
B_{O}=\sum_{i=S_{C}}^{S} P(i) \\
P_{f}^{\prime}=\sum_{k=0}^{\infty} P(S+k) P_{f h \mid k} \quad \text { and termination probability of the call is }
\end{gathered}
$$

In the FIFO handover Prioritization scheme the probability of the forced termination is decreased however the handover call may be dropped because the handover request can only wait until the receiver threshold is reached [33].

Some new queuing schemes like Measurement based prioritization scheme (MBSP), very early assignment (VEA), early assignment (EA) and most critical first (MCF) are proposed to improve the performance to improve of the handover queuing scheme by modifying the queuing discipline. In the MBSP the handover calls are added to the queue and the priorities of the calls changes dynamically based on the power level they have. The call with the power level close to the receiver threshold has the highest priority this scheme produce better results than the FIFO queuing schemes. Each of these schemes has its advantages and disadvantages in the term of capacity and services. Like the VEA gives the shortest call setup but is most capacity inefficient [20].

3.3.4 Cell Overlapping and Load Balancing Scheme (Proposed Scheme) 
In order to improve the handover call prioritization scheme it is advisable to equalize the traffic load over the cells. Traffic reason and directed retry handover make use of this principal. First the new call to be served and if the receiver is able hear a neighboring cell and are not considered in this situation. Traffic reason handover can be used to transfer traffic from one cell to another neighboring when they are closed to the congestion. The traffic reason handover idea is based on the neighboring cell having an overlapping service area. The overlapping service area arises naturally in GSM cellular system especially in small-cell high capacity micro cellular configurations. The small-cells are capture by subdividing a congested cell each with his own base station. The call arising in the common area (overlapping) of cells have access to channels more than one base station. By appropriate control strategy a cell may select the base station to establish a connation and contribute to efficient spectrum management. By subdividing a congested cell into small- cell the frequency reuse distance is effectively increased which reduce the level of interference and increase the carrier the carrier to interference ratio at both side the mobile station and base station.

Form the previous work it has been proved the directed retry an increase in the overlapping between cells leads to increases the quality of service of the cellular system. A large overlapping area gives more capacity than a smaller overlap, but even by just having a small overlap a significant gain is achieved. The overlap of $0.1 \mathrm{R}$ (where $\mathrm{R}$ is the radius of cell) results an overlapping area equal to $9 \%$ of the cell area gives a gain of at least $6 \%$ whereas if the overlap is equal to $0.5 \mathrm{R}$ means overlapping area is $75 \%$ of the cell area then the capacity gain is boost to $27 \%$. The performance of this functionality is very dependent on the existing overlapping between cells since it is required that at least one neighboring cell has sufficient signal level for the mobile station to be redirected [20].

According to the concept of cell radius when two or more adjacent cells overlap they form a set of individual regions which can be categorized into three types $\mathrm{A}, \mathrm{B}$, and $\mathrm{C}$ according to the number of cell they overlap as shown in figure 19 . These regions can be assigned a channel from one of three cells. The importance of the regions and areas is to perform the channel allocation scheme based on either through the region or area. The number of channels for specific region depends on the size of the regions and specified channel can be used in that area. If we sum of the regions in one cell according to their overlap then they formed a cell area. The blocking probability of the cell can be calculated from those users who are able of choosing a channel from cells A, B, and C. This maintains the same lowest blocking probability and load balancing in every area [16].

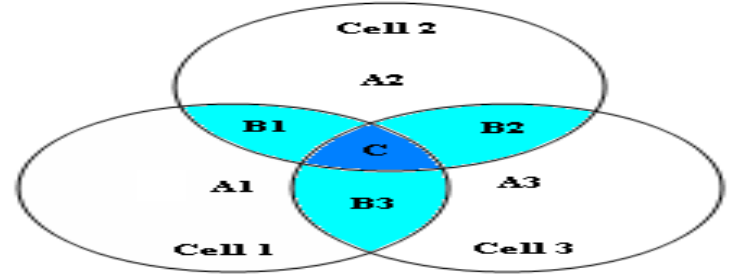

Figure 19. Areas A, B and C of three Cells

\section{ANALYSIS}

In this paper both the prioritized and the nonprioritized handover scheme are presented. Moreover different prioritization schemes and there extensive classification are presented as well. All the handover prioritization schemes allocate channels to handovers more frequently than the new call to guarantee the users QoS perspective because new calls are less sensitive to delay than the handover calls. One of the simplest ways introduced in the above literature of giving priority to the handover calls is to reserve a number of channels exclusively for the handover in each cell to improve the performance of the cellular system. The guard channel prioritization schemes are established only when the number of free channels is less or equal to predefined threshold. The value of the threshold directly affects the probability of the call blocking and call dropping. According to the cell channel occupancy by Markov chain it is straight forward to derive the steady state probability $\mathrm{P}_{\mathrm{n}}$ that $\mathrm{n}$ channels are busy and then $\mathrm{P}_{\mathrm{b}}=\sum_{\mathrm{n}=\mathrm{T}+1}^{\mathrm{C}} \quad \mathrm{P}_{\mathrm{n}}$ and $\mathrm{pf}=\mathrm{Pc}$. The equation $\mathrm{pf}=\mathrm{Pc}$ shows that the handover failure probability is equal to the call completion probability. A critical parameter is the number of channel guard channel exclusively for handover in each cell. In fact there is relationship between minimizing pd (call dropping probability) and minimizing pb (call blocking probability). The guard channel prioritization scheme has the risk of underutilizing the frequency channels or insufficient spectrum utilization. According to the equation if the number of guard channels are conservatively chosen then the admission control fails to satisfy the specified the call dropping probability. Therefore an efficient estimation method for the optimum number of guard channel is essential.

In addition when a user moves into new cell, bandwidth is reserved in the new neighboring cell and the reserved bandwidth in the cell which are no longer used to the new cell is released. However from the operation point of view there are several weaknesses in CAC scheme. First it seems difficult for CAC scheme to handle the user's request where the capacity is not enough to deal with all the requirements. In the situation the calls blocking and dropping probability increases which affects QoS of the wireless cellular network. But if the user has predefined priority then the CAC can distribute capacity according to each user so that the requirements of each with higher priority will be fulfilled prior to any other user. In such situation the CAC algorithms becomes more complicated and complex to meet with these requirements. Secondly during rush calling events such is at the end of concerts or cricket or football match or other such big events the network is flooded by the high volume of user calls. Blocking of user during such situation will help lighten the cellular network congestion but will result unsatisfied customers and lost of revenue. Furthermore some calls are blocked unnecessary in the neighboring cells due to the sudden fluctuation in the new call and the handover arrival rates. Finally as the CAC control uses the high level of frequency reuse this leads to costly implementation and more interference in the wireless cellular network.

Several other strategies to allocate channel for the handover request in the queue discipline have been proposed. For example queuing of new call arrivals is possible and is less sensitive regarding the queuing time than the case of handover. Queuing of 
the new call request shows more improvement than queuing of handover calls. In this scheme new call will be accepted if the number of free channels apart of those reserved for handover is enough for the new request otherwise the call be placed in the queue. As soon as the channel is released by the completing a call or outgoing of the handover request then the new call is served immediately from the FIFO queue. The queuing of the new calls involves the concept of the guard channels and queuing schemes. The performance analysis of queuing new call shows; that the blocking of the handover call decreases with the queuing probability of the new calls and increased in the total carried traffic because new calls will be ultimately served. This scheme also achieves less force termination probability compared to other schemes.

\section{CONCLUSION}

In this paper I introduce in greater depth the GSM network architecture and handover process which emphasizes the architecture, the several functional network elements and their dedicated channels associated with the call. Furthermore I have discussed the different performance metrics used to make the handover decision. Next I have presented the most important procedure of GSM handover initiation, handover types and their measurements reports to ensure mobility in GSM network and to emphasis the fact that handover in GSM network are very important to maintain the quality of a call.

I also investigate the call handover prioritization schemes that prioritize handover calls in order to enhance the quality of service (QOS) of GSM wireless network. Extensive survey and analysis of the handover prioritization schemes that is guard channels, call admission control and handover queuing has been provided. Furthermore my research indicates that different system uses different schemes to execute the handover mechanism for a couple of enhancements to the handover mechanism are introduced and discussed. The idea of the cells overlap and load balancing scheme which tries to equalize the traffic over cells has been introduced. It has been analyzed theoretically and mathematically that capacity depends on the size of the overlapping area between adjacent cells, the numbers of channels per cells and distribution of traffic. The higher the overlapping area, the higher the trunking efficiency gains. The overlapping area can be used to reduce the call blocking and dropping probabilities. The attractive feature of this scheme is that it organizes traffic in distributed manner and doesn't increase the system complexity. At last I conclude that the implementation of mathematical formulas as mentioned in research will be great contribution in call handoff and for QOS's.

\section{ACKNOWLEDGMENTS}

My thanks with core of my heart to IJCA to give me the opportunity to explore and share our ideas with all, I also thanks my family who support me and pray for me.

\section{REFERENCES}

[1] Abdulova, V., and Aybay, I. (2006) Handoff Prioritizing Schemes in Cellular Wireless Networks. IEEE Computer Networks Symposiums. 16 (18), pp 1-6.
[2] Ariton, E \& Xhafa. (2005) Dynamic Priority Queuing of Handover Calls in Wireless Network: An Analytical Framework. 22(5), June 2004, pp. 904- 916.

[3] Sudhir Agrawal,Sarvpal H. Singh,C. Ramakrishna(2010) Location Management Optimization Schemes for Cellular System

http://www.ijcaonline.org/volume4/number4/pxc3871162.pdf

[4] Cello. (2001)Cellular Network Optimization Based on Mobile Location. [Internet]. Finland. Available from $:<$ http://www.telecom.ntua.gr/cello/ > [Accessed 20 Jan 2007].

[5] Chaiko, Y. (2004) Simulation of Radio wave Propagation using Propagation Models. [Internet].Available from: <:http://www.tmf.rtu.lv/DATA_FILES/blank_sc_publication/6/1 001/34/2005_03_02_10_34_11/paper_formatting_Caiko.doc> [Accessed 6 Jan 2007].

[6] Dirk, H. (2002) The GSM Radio Interface. [Internet]. Available from $:<$ http://www.aws.cit.ie/personnel/dpesch/notes/msc_sw/GSM_radi o_interface.pdf $>\quad$ [Accessed 4 Jan 2007].

[7] Ekici, E. (1997) Optimal Two-Tier Cellular Network Design. BS Dissertation, Bogazici University, Turkey.

[8] Farely, T. (2006) Early Bell System Overview AMPS [Internet]. USA, Available from :< http://www.privateline.com/mt_cellbasics/x_appendix/c_early_b ell_system_overview_of_amps/>[Accessed 17 Sep 2006].

[9] Fournogerakis, P., Kyriazakos, S., \& Karetsos, G. (2001) Enhanced Handover Performance in Cellular Systems based on Position Location of Mobile Terminals. [Internet]. Athens, Greece. Available from :< http://www.telecom.ece.ntua.gr/cello/documents/CELLO-WP5ICCS-PUB02-013-Int.pdf > [Accessed 9 Jan 2007].

[10] Ghaderi, M and Boutaba. (2006) Call Admission Control in Mobile Cellular Networks: A Comprehensive Survey. Wireless Communications and Mobile Computing, 6(1), pp 69-93. Available from http://bcr2.uwaterloo.ca/ rboutaba/Papers/Journals/WCMC04.pdf> [Accessed 9 March 2007].

[11] Harmantzis, C \&Yaipairoj, S. (2005) Dynamic Pricing with Alternatives for Mobile Network. IEEE Personal Communications. [Internet]. Available from $:<$ http://personal.stevens.edu/ syaipair/A28_1.pdf> [Accessed 10 May 2007].

[12] IEEE, Handover Schemes in Satellite Networks: State-OfThe-Art and Future Research Directions, NASA, 2006, Volume $8(4)$.

[13] 1CH. M.H. Saibaba 2Prof. Ravindra Babu Kallam 2Dr. M. ThirupathiReddy (2010) Eradication of Magnetic Radiations Produced By Mobiles Devices http://www.ijcaonline.org/journal/number7/pxc387278.pdf

[14] International Engineering Construction. (2005) Cellular Communications. [Internet]. USA, IEC. Available from :< http://www.iec.org/online/tutorials/cell_comm/topic03.html > [Accessed 14 Sep 2006]. 
[15] Katzela, I and Naghshineh. (1996) Channel Assignment Scheme for Cellular Mobile Telecommunication Systems- A Comprehensive Survey, IEEE Personal Communications, 3 (3): pp.10-31.

[16] Katzis, K. (2005) Resource Allocation Techniques for High Altitude Platforms. PhD Dissertation, University of York, UK.

[17] Kriegl, J. (2000) Location in Cellular Networks. Diploma Thesis, University of Technology Graz, Australia.

[18] Li, J., Shorff, N and Chong, E (1996) Channel Carrying: A Novel Handoff Scheme for Mobile Cellular Networks. [Online]. ECE Technical Reports, Available from: < http://docs.lib.purdue.edu/ecetr/98/> [Accessed 6 March 2007]. [19] Mandjes, M., and Tutschku, K. (1996) Efficient Call Handling Procedures in Cellular Mobile Networks, [Online].Report No.144, Available from :< http://www3.informatik.uni-

wuerzburg.de/publications/reports.shtml > [Accessed 4 March 2007].

[20] Nielsen, T., and Wigard, J. (2000) Performance Enhancements in a Frequency Hopping GSM Network. Kluwer Academic Publisher, Netherlands.

[21] Nishitd, D., Jeffrey, N and Vanlandingham, F. (1998) Handoff in Cellular Systems. IEEE Personal Communications. [Internet]. Available from :< http://www.comsoc.org/pci/private/1998/dec/Tripathi.html> [Accessed 10 March 2006].

[22] Reyaz, W. (2003) GSM-GPRS-EDGE Technologies. Master Thesis, Department of Computer Engineering Indian Institute of Technology, New Delhi. India.

[23] Scourias, J. (1997) Overview of Global System for Mobile Communications. [Internet]. Available from :< http://www.shoshin.uwaterloo.ca/ jscouria/GSM/gsmreport.html $>$ [Accessed 26 Sep 2006].

[24] Soy, S. (1997) The Case Study as a Research Method. [Internet]. Available from: http://www.gslis.utexas.edu/ ssoy/pubs/index.html> [Accessed 9 April 2007].

[25] Stallings, W. (2005) Wireless Communications \& Networks. $2^{\text {nd }}$ Ed. USA, Prentice Hall.
[26] Thomsen, J., and Manggard, R. (2003) Analysis of GSM Handover using Coloured Petri Nets. Master Thesis, University of Aarhus, Denmark.

[27] Tripathi, N., Nortel, Jerry, R and Vanlandingham. (1998) Handoff in Cellular Systems. [Internet] .IEEE Personal Communications. Available from $<$ https://www.cs.tcd.ie/ htewari/papers/tripathi98.pdf> [Accessed 7 March 2007].

[28] Wei, Y. (2001) Evaluation of Roaming and Download Times in Universal Cellular/Wireless LAN System. Master Thesis Dissertation. Concordia University, Canada.

[29] Wikipedia. (2007) Case Study. [Internet].US. Available from : $<$ http://en.wikipedia.org/wiki/Case_studies > [Accessed 20 April 2007].

[30] Wikipedia. (2007) Qualitative Research[Internet].Available from :< http://en.wikipedia.org/wiki/Qualitative_method > [Accessed 3 April 2007].

[31] Willig, A. (2003) The GSM Air Interface Fundamental and Protocols. [Internet].Available from :< http://www.dcl.hpi.uni-

potsdam.de/teaching/mobilitySem03/slides/hpi_gsm_air_interfac e.pdf > [Accessed 3 Jan 2007].

[32] Yliopisto, O. (2005) Smart Packet and Call Admission Control for Efficient Resource Management in Advanced Wireless Networks. PhD Dissertation. University of Oulu, Finland.

[33] Zeng, Q., Agrawal, D. (2001) Handoff in Wireless Mobile Networks. [Internet].Ohio.USA. Available from :< http://media.wiley.com/product_data/excerpt/28/04714190/04714 19028.pdf> [Accessed 20 Jan 2007].

[34] Zhang, J. (2005) Cellular Networks [Internet]. Available from:

<http://www.site.uottawa.ca/ ivan/cellular.pdf > [Accessed 17 Sep 2006]. 